\title{
Patient outcomes and prognostic factors associated with colonic perforation surgery: a retrospective study
}

\author{
Do-bin Lee, Seonhui Shin, Chun-Seok Yang \\ Department of Surgery, Daegu Catholic University Medical Center, Daegu Catholic University School of Medicine, Daegu, Korea
}

Background: Despite advances in surgery and intensive perioperative care, fecal peritonitis secondary to colonic perforation is associated with high rates of morbidity and mortality. This study was performed to review the outcomes of patients who underwent colonic perforation surgery and to evaluate the prognostic factors associated with mortality.

Methods: A retrospective analysis was performed on 224 consecutive patients who underwent emergency colonic perforation surgery between January 2008 and May 2019. We divided the patients into survivor and non-survivor groups and compared their surgical outcomes.

Results: The most common cause of colon perforation was malignancy in 54 patients $(24.1 \%)$, followed by iatrogenic perforation in $41(18.3 \%)$, stercoral perforation in $39(17.4 \%)$, and diverticulitis in $37(16.5 \%)$. The sigmoid colon $(n=124,55.4 \%)$ was the most common location of perforation, followed by the ascending colon, rectum, and cecum. Forty-five patients $(20.1 \%)$ died within 1 month after surgery. Comparing the 179 survivors with the 45 non-survivors, the patient characteristics associated with mortality were advanced age, low systolic blood pressure, tachycardia, organ failure, high C-reactive protein, high creatinine, prolonged prothrombin time, and high lactate level. The presence of free or feculent fluid, diffuse peritonitis, and right-sided perforation were associated with mortality. In multivariate analysis, advanced age, organ failure, right-sided perforation, and diffuse peritonitis independently predicted mortality within 1 month after surgery.

Conclusion: Age and organ failure were prognostic factors for mortality associated with colon perforation. Furthermore, right-sided perforation and diffuse peritonitis demonstrated a significant association with patient mortality.

Keywords: Intestinal perforation; Peritonitis; Postoperative complication; Surgical stomas

\section{Introduction}

Fecal peritonitis secondary to colonic perforation is a life-threatening condition, and emergency surgical management is associated with high morbidity and mortality rates. Despite advances in surgical techniques and perioperative management, surgical outcomes after colonic perforation have not improved [1-4]. It is important to preoperatively evaluate the severity of peritonitis and identify the associated risk factors for mortality because patients with severe peritonitis require immediate surgical management and high-quality intensive care. Additionally, the severity of the peritonitis, potential predictors, and the likelihood of mortality should be included in the information provided to patients and their families.

Several studies have investigated the prognostic factors associated with the mortality and morbidity of patients with fecal peritonitis; consequently, several scoring systems are available [1,5-7].

Received: August 11, 2021 • Revised: September 18, 2021 • Accepted: September 28, 2021

Corresponding author: Chun-Seok Yang, MD

Department of Surgery, Daegu Catholic University Medical Center, Daegu Catholic University School of Medicine, 33 Duryugongwon-ro 17-gil, Nam-gu, Daegu 42472, Korea

Tel: +82-53-650-4065• Fax: +82-53-624-7185•E-mail: gsyangcs@gmail.com 
However, these risk factors and scoring systems have only been validated in small-population studies and are not clinically prevalent. Nonetheless, many studies are ongoing to determine other risk factors associated with mortality in patients who undergo colonic perforation.

Hartmann's procedure is the standard surgical procedure for treating left colonic perforation [8,9]. Hartmann's procedure is associated with low quality of life because of the colostomy care required [10], and restoration of intestinal continuity is associated with morbidity and mortality [11]. The reversal rate of Hartmann's procedure tends to be lower than 50\% in most reported articles [12]. However, it is inconclusive whether primary resection and anastomosis without fecal diversion is safer than Hartmann's procedure for left colonic perforation.

Thus, the aim of this observational retrospective study was to review the outcomes of patients who underwent emergency surgery for fecal peritonitis secondary to colonic perforation. The primary objective was to compare various factors between survivors and non-survivors. Accordingly, we aimed to predict outcomes based on patient conditions before and during surgery. The secondary objective was to evaluate the necessity of fecal diversion in patients with left colonic perforation without prognostic factors.

\section{Methods}

Ethical statements: The study protocol was approved by the Institutional Review Board (IRB) of Daegu Catholic University Hospital (IRB No: CR-21-069), which waived the need for informed consent due to the retrospective design of the study.

\section{Study design and population}

For this retrospective study, we selected 224 consecutive patients who underwent emergency surgery for fecal peritonitis secondary to colon perforation between January 2008 and May 2019 at Daegu Catholic University Hospital in Korea. Patients with perforations from other gastrointestinal conditions were excluded.

\section{Data collection}

Patient clinical and management data were collected from medical chart reviews. This included patient demographics such as sex, age, height, weight, body mass index, comorbidities, time from symptom onset to surgery, American Society of Anesthesiologists (ASA) physical status (PS) classification, initial vital signs, white blood cell count, hemoglobin, prothrombin time, activated partial thromboplastin time, serum protein levels, albumin, lactate, C-reactive protein (CRP), creatinine, and blood urea nitrogen at the time of admission. The surgical and pathological reports were reviewed to obtain data regarding the extent of peritoneal contamination, the types of surgery performed, and the sites and causes of perforation. In addition, the length of operation, perioperative complications, mortality, and length of hospital stay were reviewed. Patients were divided into survivors and non-survivors, and clinical data were compared between the groups.

We defined advanced age as $>70$ years. Organ failure was defined as follows: (1) renal failure with creatinine levels of $>1.4$ $\mathrm{mg} / \mathrm{dL},(2)$ circulatory failure with systolic arterial pressure of $<90 \mathrm{mmHg}$ requiring inotropes, and (3) respiratory failure with partial pressure of oxygen $<60 \mathrm{mmHg}$. Free fluid was defined as the presence of hypodense fluid within the pelvic cavity, subphrenic space, paracolic gutters, or other intraperitoneal spaces on computed tomography (CT) scan. Feculent fluid was defined as the presence of feces protruding through the perforation site with spillage to the adjacent peritoneal cavity on CT scan. Free perforation was defined as air bubbles or air collection within the abdominal cavity, with a distance greater than $5 \mathrm{~cm}$ of the affected bowel segment. Diffuse peritonitis was defined as contamination or exudate in the four quadrants during surgery.

The left colon was defined as the descending colon to the rectum. Perioperative mortality was defined as death occurring within 1 month of surgery. Complications were graded according to the Clavien-Dindo classification [13].

\section{Operative parameters}

We reported postoperative morbidity and mortality, fecal diversion, specialty of the attending surgeon, and rate of bowel reconstruction. The operation results (Hartmann's procedure, primary resection and anastomosis with or without fecal diversion, anastomotic dehiscence, or stoma closure) were also recorded.

\section{Subgroup analysis}

In accordance with our second objective, we evaluated the necessity of fecal diversion in patients with left colonic perforation without prognostic factors. Thus, to compare the surgical outcomes with or without fecal diversion, we excluded 106 patients who had diffuse peritonitis from the 165 patients with left colonic perforation because diffuse peritonitis was an independent surgical prognostic factor. Thus, the sub-analysis included a group of patients with left colonic perforation at low risk of mortality. Accordingly, 59 patients were included in the sub-analysis.

\section{Statistical methods}

Comparisons between groups were performed using the chi- 
square test or Student $t$-test. We used two-way contingency tables to compare discrete variables and implemented Fisher exact test when low expected values were present. Univariate and multivariate analyses were performed. The variables were analyzed for various outcomes using simple logistic regression, and odds ratios and $95 \%$ confidence intervals were reported. For multivariate analysis, a multiple logistic regression model was used. The Mann-Whitney $U$-test was used to analyze the differences in non-categorical variables among the subgroups. All analyses were performed using IBM SPSS version 21.0 (IBM Corp., Armonk, NY, USA) and all $p$-values were two-sided; $p$-values of $<0.05$ were considered statistically significant.

\section{Results}

Two hundred and twenty-four patients were included in this study. The patient characteristics are presented in Table 1 . The mean patient age was $67.5 \pm 15.3$ years ( 102 male, 122 female). The most common perforation site was the sigmoid colon in 124 patients (55.4\%); 59 patients (26.3\%) had perforations on the right side and 165 (73.7\%) had perforations on the left side. Resection and anastomosis (50.0\%) were the most frequently performed surgeries, followed by Hartmann's procedure (27.7\%). Malignancy was the most common cause of perforation in both groups, but stercoral and ischemic colitis were more common in the non-survivors.

A comparison of the factors associated with survivors and non-survivors indicated that age ( $66 \pm 15.5$ years vs. $73.4 \pm 13.2$ years, $p=0.004)$, organ failure ( $19.6 \%$ vs. $53.3 \%, p<0.001)$, systolic blood pressure $(117.7 \pm 22 \mathrm{mmHg}$ vs. $105.4 \pm 30.3 \mathrm{mmHg}$, $p=0.003)$, and heart rate $(87.5 \pm 16.1$ beats/min vs. $94.5 \pm 20.6$ beats $/ \mathrm{min}, p=0.041$ ) were significantly different (Table 2 ). Analysis of laboratory values indicated that CRP, creatinine, prothrombin time, and lactate levels were significantly different between the groups. Analysis of the factors associated with characteristics of peritonitis indicated that free fluid ( $50.6 \%$ vs. $76.7 \%, p=0.003$ ), feculent fluid ( $36 \%$ vs. $55.6 \%, p=0.027$ ), diffuse peritonitis ( $52.6 \%$ vs. $82.2 \%, p=0.001)$, and right-sided perforation $(22.9 \%$ vs. $40 \%, p=0.033)$ were significantly different. The operative outcomes are summarized in Table 3. There were 89 patients (39.7\%) with Clavien-Dindo classification $\geq$ III, of whom 45 (20.1\%) died within 1 month after surgery. Of the total, 120 patients (53.6\%) underwent fecal diversions, such as Hartmann's procedure, ileostomy, or colostomy, and 64 (72.7\%) of the 88 surviving patients underwent stoma closure.

The univariate and multivariate regression analyses are presented in Table 4 . Age of $>70$ years, $\geq 2$ comorbidities, organ failure, renal failure, prolonged prothrombin time, free fluid, feculent fluid, diffuse peritonitis, and right-sided perforation were associated with mortality. When multivariate analysis was performed to determine whether the aforementioned factors were prognostic, only advanced age, organ failure, right-sided perforation, and diffuse peritonitis were statistically significant.

Sub-analysis between the group that underwent fecal diversion $(\mathrm{n}=30)$ and the group that underwent surgical methods without fecal diversion $(n=29)$ revealed that there was no significant difference between the groups in terms of preoperative and intraoperative findings such as age and comorbidities. This suggests that there were no preoperative differences in other risk factors for mortality. Moreover, there were nine patients $(30.0 \%)$ whose stoma could not be reversed. Nonetheless, there was no significant difference in mortality and morbidity between the groups (Table 5).

\section{Discussion}

Colonic perforation causes widespread dissemination of bacteria and feces into the intraperitoneal space and can lead to peritonitis, septic shock, and multiple organ failure. The mortality rate associated with colon perforation reportedly ranges from $6.2 \%$ to $33.3 \%$ [1,5-7,14-17]. Similarly, the mortality rate in our study was $20.1 \%$. Our results also showed that old age, organ failure, right-sided perforation, and diffuse peritonitis were associated with mortality. In addition, in the absence of diffuse peritonitis, even patients with left colon perforations showed comparable surgical outcomes with or without fecal diversion. Thus, considering that there are several complications associated with stoma reversal and that many patients have a permanent stoma, a stoma can be omitted if there are no associated risk factors.

Fecal peritonitis due to colonic perforation is largely associated with mortality due to factors such as patient characteristics (including age, ASA PS classification, concurrent medical disease, immunosuppression, and performance status), peritonitis severity, or an interaction between them. Factors such as organ failure and various deteriorations of the blood represent an interaction between patient characteristics and peritonitis severity. Moreover, there are reports of worsening prognosis in patients with lactic acidosis, leukopenia, and bleeding tendency $[9,18,19]$.

In a large cohort of patients with fecal peritonitis, the strongest independent risk factors for mortality were increased age, renal dysfunction, hypothermia, and low hematocrit levels [20]. Furthermore, Tan et al. [21] showed that there was a significant association between mortality and morbidity rates and ASA PS classification, as well as the acute physiology component of the Acute Physiology and Chronic Health Evaluation II score in patients 
Table 1. Characteristics of the study population and comparison between the survivors and non-survivors

\begin{tabular}{|c|c|c|c|c|}
\hline Characteristic & Overall & Survivor & Non-survivor & $p$-value \\
\hline No. of patients & 224 & 179 & 45 & \\
\hline Sex & & & & $>0.999$ \\
\hline Male & $102(45.5)$ & $82(45.8)$ & $20(44.4)$ & \\
\hline Female & $122(54.5)$ & $97(54.2)$ & $25(55.6)$ & \\
\hline Age (yr) & $67.5 \pm 15.3$ & $66.0 \pm 15.5$ & $73.4 \pm 13.2$ & 0.004 \\
\hline ASA PS classification & & & & 0.118 \\
\hline 1 & $52(23.2)$ & $47(26.3)$ & $5(11.1)$ & \\
\hline$\|$ & $119(53.1)$ & $94(52.5)$ & $25(55.6)$ & \\
\hline III & $49(21.9)$ & $35(19.6)$ & $14(31.1)$ & \\
\hline IV & $4(1.8)$ & $3(1.7)$ & $1(2.2)$ & \\
\hline Location & & & & 0.160 \\
\hline Cecum & $18(8.0)$ & $14(7.8)$ & $4(8.9)$ & \\
\hline Ascending colon & $22(9.8)$ & $16(8.9)$ & $6(13.3)$ & \\
\hline Hepatic flexure colon & $3(1.3)$ & $2(1.1)$ & $1(2.2)$ & \\
\hline Transverse colon & $16(7.1)$ & $8(4.5)$ & $7(15.6)$ & \\
\hline Splenic flexure colon & $5(2.2)$ & $4(2.2)$ & $2(4.4)$ & \\
\hline Descending colon & $15(6.7)$ & $12(6.7)$ & $3(6.7)$ & \\
\hline Sigmoid colon & $124(55.4)$ & $105(58.7)$ & $19(42.2)$ & \\
\hline Rectum & $21(9.4)$ & $18(10.1)$ & $3(6.7)$ & \\
\hline Sidedness & & & & 0.033 \\
\hline Right location & $59(26.3)$ & $41(22.9)$ & $18(40.0)$ & \\
\hline Left location & $165(73.7)$ & $138(77.1)$ & $27(60.0)$ & \\
\hline Types of surgery & & & & 0.004 \\
\hline Hartmann's operation & $62(27.7)$ & $41(22.9)$ & $21(46.7)$ & \\
\hline Resection and anastomosis & $112(50)$ & $93(52)$ & $19(42.2)$ & \\
\hline Primary closure & $35(15.6)$ & $33(18.4)$ & $2(4.4)$ & \\
\hline Stoma only & $11(4.9)$ & $10(5.6)$ & $1(2.2)$ & \\
\hline Drainage only & $4(1.8)$ & $2(1.1)$ & $2(4.4)$ & \\
\hline Stoma creation & 120 (53.6) & $88(49.2)$ & $32(71.1)$ & 0.013 \\
\hline No stoma creation & $104(46.4)$ & $91(50.8)$ & $13(28.9)$ & \\
\hline Causes of perforation & & & & 0.023 \\
\hline Malignancy & $54(24.1)$ & $41(22.9)$ & $13(28.9)$ & \\
\hline Diverticulitis & $37(16.5)$ & $30(16.8)$ & $7(15.6)$ & \\
\hline latrogenic & $41(18.3)$ & $39(21.8)$ & $2(4.4)$ & \\
\hline Ischemic colitis & $16(7.1)$ & $9(5.0)$ & $7(15.6)$ & \\
\hline Stercoral & $39(17.4)$ & $28(15.6)$ & $11(24.4)$ & \\
\hline Trauma & $24(10.7)$ & $21(11.7)$ & $3(6.7)$ & \\
\hline Unknown & $13(5.8)$ & $11(6.1)$ & $2(4.4)$ & \\
\hline
\end{tabular}

Values are presented as number only, number (\%), or mean \pm standard deviation. ASA, American Society of Anesthesiologists; PS, physical status.

with colon cancer perforation. These findings are further supported by those of Yoo et al. [22]. Thus, a scoring system is useful for objectively describing patient conditions, thereby aiding surgical decisions for patients with fecal peritonitis [5].

While the predictive value of factors that reflect the severity of peritonitis has been previously studied, it is difficult to quantitatively measure the degree of peritonitis. Until now, only a scoring system based on the peritonitis scope and spillage content has been developed. Nevertheless, these studies emphasize the importance of diffuse peritonitis. There are reports that the spread of ascites on a preoperative $\mathrm{CT}$ scan is significant for predicting mortality [18]. Similarly, we showed that prognosis was not affected by the degree of contamination of the ascites, but rather the extent to which it had spread. These results support the speculation that the 
Table 2. Comparison of perioperative factors between the survivors and non-survivors

\begin{tabular}{|c|c|c|c|}
\hline Characteristic & Survivor $(n=179)$ & Non-survivor $(n=45)$ & $p$-value \\
\hline Time from symptom onset to surgery (day) & $1.6 \pm 2.7$ & $2.2 \pm 3.6$ & 0.251 \\
\hline Organ failure & $35(19.6)$ & 24 (53.3) & $<0.001$ \\
\hline Systolic blood pressure $(\mathrm{mmHg})$ & $117.7 \pm 22$ & $105.4 \pm 30.3$ & 0.003 \\
\hline Heart rate (beats/min) & $87.5 \pm 16.1$ & $94.5 \pm 20.6$ & 0.041 \\
\hline White blood cell count $\left(10^{3} / \mu \mathrm{L}\right)$ & & & 0.178 \\
\hline$<4.0$ & $30(16.8)$ & $13(28.9)$ & \\
\hline$\geq 4.0,<10.0$ & $73(40.8)$ & $15(33.3)$ & \\
\hline$\geq 10.0$ & $76(42.5)$ & 17 (37.8) & \\
\hline C-reactive protein (mg/L) & $89.7 \pm 104.4$ & $135.2 \pm 119.1$ & 0.029 \\
\hline Creatinine $(\mathrm{mg} / \mathrm{dL})$ & $1.1 \pm 1.1$ & $1.4 \pm 0.9$ & 0.037 \\
\hline Prothrombin time (sec) & $14.4 \pm 1.4$ & $16.3 \pm 3.1$ & $<0.001$ \\
\hline Lactate (mmol/L) & $2.8 \pm 1.9$ & $5.5 \pm 2.4$ & 0.001 \\
\hline Free perforation & $142(81.1)$ & $40(88.9)$ & 0.315 \\
\hline Free fluid & $88(50.6)$ & $33(76.7)$ & 0.003 \\
\hline Feculent fluid & $63(36.0)$ & $25(55.6)$ & 0.027 \\
\hline Abscess & $23(13.1)$ & $4(9.3)$ & 0.670 \\
\hline Diffuse peritonitis & $92(52.6)$ & $37(82.2)$ & 0.001 \\
\hline Retroperitoneal perforation & $25(14.3)$ & 7 (15.9) & 0.973 \\
\hline
\end{tabular}

Values are presented as mean \pm standard deviation or number (\%).

Table 3. Comparison of operative outcomes between the survivors and non-survivors

\begin{tabular}{lccc}
\hline Characteristic & Survivor $(\mathrm{n}=179)$ & Non-survivor $(\mathrm{n}=45)$ & $p$-value \\
\hline Colorectal surgeon & $137(76.5)$ & $30(66.7)$ & 0.243 \\
Operative time (min) & $169.5 \pm 67.1$ & $186.2 \pm 131.1$ & 0.235 \\
Intraoperative lavage & $14(7.8)$ & $2(4.4)$ & 0.745 \\
Stoma reversal & $64(72.7)^{a)}$ & & NA \\
Complication & & & \\
$\quad$ Surgical site infection & $47(26.3)$ & $10(22.2)$ & 0.716 \\
Intraabdominal abscess & $36(20.1)$ & $8(17.8)$ & 0.887 \\
Septic shock & $9(5.0)$ & $40(88.9)$ & $<0.001$ \\
Pneumonia & $19(10.6)$ & $8(17.8)$ & 0.288 \\
Cardiovascular events & $3(1.7)$ & $13(28.9)$ & $<0.001$ \\
Paralytic ileus & $8(4.5)$ & $0(0)$ & 0.363 \\
Anastomosis leakage & $2(1.1)$ & $6(13.3)$ & 0.001 \\
$\quad$ Multiorgan failure & $1(0.6)$ & $39(86.7)$ & $<0.001$ \\
Clavien-Dindo classification & & & NA \\
I & $19(10.6)$ & & \\
II & $58(32.4)$ & & \\
III & $38(21.2)$ & & \\
IV & $6(3.4)$ & & \\
\hline
\end{tabular}

Values are presented as number (\%) or mean \pm standard deviation.

NA, not applicable.

${ }^{\text {a) }}$ The stoma reversal rate was based on 88 survivors among patients with stoma creation.

spread of ascites indicates the severity of peritonitis or the duration from the onset of perforation. While mortality rates have been shown to increase with the interval length between the time of hollow organ perforation and the time of surgery [23], our results did not reveal interval length as a prognostic factor for mortality.

Furthermore, our result that diffuse peritonitis is an important prognostic factor is similar to results from studies on colorectal cancer obstruction. When perforation occurs proximal to the ob- 
Table 4. Analysis of mortality

\begin{tabular}{|c|c|c|c|c|}
\hline \multirow{2}{*}{ Variable } & \multicolumn{2}{|c|}{ Univariate analysis } & \multicolumn{2}{|c|}{ Multivariate analysis } \\
\hline & Odds ratio $(95 \% \mathrm{Cl})$ & $p$-value & Odds ratio $(95 \% \mathrm{Cl})$ & $p$-value \\
\hline \multicolumn{5}{|l|}{ Age (yr) } \\
\hline$\leq 70$ & 1 & & & \\
\hline$>70$ & $3.496(1.667-7.331)$ & 0.001 & $2.993(1.254-7.139)$ & 0.013 \\
\hline Comorbidity, $\geq 2$ & $3.032(1.549-5.934)$ & 0.001 & $1.738(0.767-3.938)$ & 0.185 \\
\hline Organ failure & $4.702(2.353-9.397)$ & $<0.001$ & $4.207(1.326-13.347)$ & 0.015 \\
\hline Renal failure & $3.568(1.663-7.658)$ & 0.001 & $0.606(0.168-2.189)$ & 0.445 \\
\hline Prolonged prothrombin time (>15 sec) & $3.225(1.646-6.319)$ & 0.001 & $1.899(0.855-4.219)$ & 0.115 \\
\hline Free fluid & $3.225(1.497-6.947)$ & 0.003 & $1.404(0.542-3.633)$ & 0.485 \\
\hline Feculent fluid & $2.222(1.144-4.317)$ & 0.018 & $0.935(0.39-2.245)$ & 0.881 \\
\hline Diffuse peritonitis & $4.173(1.838-9.472)$ & 0.001 & $3.184(1.208-8.397)$ & 0.019 \\
\hline \multicolumn{5}{|l|}{ Sidedness } \\
\hline Right location & $2.244(1.125-4.477)$ & 0.022 & $2.348(1.026-5.373)$ & 0.043 \\
\hline Left location & 1 & & & \\
\hline
\end{tabular}

$\mathrm{Cl}$, confidence interval.

Table 5. Subgroup analysis of patients with left-sided colon perforation and low risk factors

\begin{tabular}{|c|c|c|c|}
\hline Characteristic & Without fecal diversion $(n=29)$ & With fecal diversion $(n=30)$ & $p$-value \\
\hline Sex & & & 0.908 \\
\hline Male & $13(44.8)$ & $13(43.3)$ & \\
\hline Female & $16(55.2)$ & $17(56.7)$ & \\
\hline Age (yr) & $62.5 \pm 16.3$ & $65.4 \pm 16.3$ & 0.299 \\
\hline Systolic blood pressure $(\mathrm{mmHg})$ & $124.7 \pm 14.5$ & $119.2 \pm 19.2$ & 0.114 \\
\hline Heart rate (beats/min) & $85.6 \pm 14.4$ & $88.5 \pm 12.4$ & 0.346 \\
\hline C-reactive protein (mg/L) & $51.9 \pm 71.2$ & $85.2 \pm 88.7$ & 0.115 \\
\hline Creatinine (mg/dL) & $0.7 \pm 0.2$ & $0.8 \pm 0.3$ & 0.169 \\
\hline Prothrombin time (s) & $13.7 \pm 0.8$ & $14.1 \pm 1.1$ & 0.179 \\
\hline Lactate $(\mathrm{mmol} / \mathrm{L})$ & $2.2 \pm 1.0$ & $1.7 \pm 0.6$ & 0.402 \\
\hline Free perforation & $22(78.6)$ & $19(65.5)$ & 0.273 \\
\hline Free fluid & $10(35.7)$ & $9(31.0)$ & 0.708 \\
\hline Feculent fluid & $6(21.4)$ & $8(27.6)$ & 0.589 \\
\hline Abscess & $6(21.4)$ & $5(17.2)$ & 0.689 \\
\hline Retroperitoneal perforation & $6(21.4)$ & $4(13.8)$ & $0.504^{\text {a) }}$ \\
\hline Operative time (min) & $166.2 \pm 59.5$ & $185.1 \pm 63.6$ & 0.180 \\
\hline Hospital stay (day) & $13.9 \pm 6.1$ & $20.1 \pm 12.1$ & 0.050 \\
\hline Clavien-Dindo classification & & & 0.543 \\
\hline I & $1(3.4)$ & $3(10.0)$ & \\
\hline$\|$ & $10(34.5)$ & $7(23.3)$ & \\
\hline III & $10(34.5)$ & $7(23.3)$ & \\
\hline IV & $5(17.2)$ & $7(23.3)$ & \\
\hline Mortality & $2(6.9)$ & $2(6.7)$ & $>0.999^{\mathrm{a})}$ \\
\hline
\end{tabular}

Values are presented as number (\%) or mean \pm standard deviation.

${ }^{a)}$ Statistical significance was assessed by Fisher exact test.

structing tumor, it tends to be severe due to intestinal distension, and peritoneal contamination is diffuse and fecal. This leads to severe septic shock, which increases the risk of perioperative mortali- ty. In contrast, when perforation occurs at the cancer site, the contamination is usually localized, typically leading to purulent collection and resulting in a lower risk of severe peritonitis [24]. In our 
study, this is also the reason that peritonitis on the right side had a worse prognosis than that on the left side. In cases of perforation on the right side, more diffuse peritonitis occurred, which led to a worse prognosis.

The optimal surgical treatment for colonic perforation remains controversial, and the treatment strategy depends on the patient's general condition and the experience of the primary surgeon. Despite advancements in surgical techniques, Hartmann's procedure is still frequently performed to treat left colonic perforation $[8,9]$. The literature that primary anastomosis has comparable surgical outcomes to Hartmann's procedure has mainly been studied in patients with diverticular perforation [25]. Primary anastomosis without fecal diversion has a longer operative time, and Hartmann's procedure is a safer option for patients with severe medical illness. Therefore, it is important to determine the patient's overall condition and account for risk factors and conditions that are associated with mortality prior to surgery. In the absence of diffuse peritonitis, primary anastomosis and stoma omission had similar operative outcomes to Hartmann's procedure even in patients with left colon perforation.

Our study has some limitations. First, this was a single-center study, and we could not proceed with the validation process. Second, our study had a retrospective design; thus, we could not obtain some patient information, such as CRP and lactate levels. Third, because this study included patients who underwent surgery, there may be limitations in the clinical course of fecal peritonitis. Finally, the surgeries were performed by 10 different surgeons, which may have led to inconsistent quality. However, there was no significant difference in the postoperative outcomes based on the surgeon's specialty.

In conclusion, advanced age, organ failure, right-sided perforation, and diffuse peritonitis were found to be prognostic factors for colon perforation accompanied by fecal peritonitis. Thus, these findings demonstrate that it is important to determine the type of surgery, extent of resection, and whether fecal diversion should be performed.

\section{Notes}

\section{Conflicts of interest}

No potential conflict of interest relevant to this article was reported.

\section{Funding}

None.

\section{Author contributions}

Conceptualization, Data curation: all authors; Investigation: DL,
SS; Formal analysis, Project administration, Software, Supervision, Validation: CSY; Methodology: SS, CSY; Writing-original draft: DL; Writing-review \& editing: CSY.

\section{ORCID}

Do-bin Lee, https://orcid.org/0000-0002-7249-7074

Seonhui Shin, https://orcid.org/0000-0003-4676-9037

Chun-Seok Yang, https://orcid.org/0000-0002-5527-6819

\section{References}

1. Bielecki K, Kamiński P, Klukowski M. Large bowel perforation: morbidity and mortality. Tech Coloproctol 2002;6:177-82.

2. Tridente A, Bion J, Mills GH, Gordon AC, Clarke GM, Walden $A$, et al. Derivation and validation of a prognostic model for postoperative risk stratification of critically ill patients with faecal peritonitis. Ann Intensive Care 2017;7:96.

3. Biondo S, Kreisler E, Millan M, Fraccalvieri D, Golda T, Martí Ragué J, et al. Differences in patient postoperative and longterm outcomes between obstructive and perforated colonic cancer. Am J Surg 2008;195:427-32.

4. Lee IK, Sung NY, Lee YS, Lee SC, Kang WK, Cho HM, et al. The survival rate and prognostic factors in 26 perforated colorectal cancer patients. Int J Colorectal Dis 2007;22:467-73.

5. Biondo S, Ramos E, Deiros M, Ragué JM, De Oca J, Moreno P, et al. Prognostic factors for mortality in left colonic peritonitis: a new scoring system. J Am Coll Surg 2000;191:635-42.

6. Shinkawa H, Yasuhara H, Naka S, Yanagie H, Nojiri T, Furuya Y, et al. Factors affecting the early mortality of patients with nontraumatic colorectal perforation. Surg Today 2003;33:13-7.

7. Horiuchi A, Watanabe Y, Doi T, Sato K, Yukumi S, Yoshida M, et al. Evaluation of prognostic factors and scoring system in colonic perforation. World J Gastroenterol 2007;13:3228-31.

8. Cirocchi R, Trastulli S, Desiderio J, Listorti C, Boselli C, Parisi A, et al. Treatment of Hinchey stage III-IV diverticulitis: a systematic review and meta-analysis. Int J Colorectal Dis 2013; 28:447-57

9. Joo Y, Lee Y, Yoo T, Kim J, Park I, Gwak G, et al. Prognostic factors and management for left colonic perforation: can Hartmann's procedure be preventable? Ann Coloproctol 2020;36: 178-85.

10. Vermeulen J, Gosselink MP, Busschbach JJ, Lange JF. Avoiding or reversing Hartmann's procedure provides improved quality of life after perforated diverticulitis. J Gastrointest Surg 2010; 14:651-7.

11. Richards CH, Roxburgh CS; Scottish Surgical Research Group (SSRG). Surgical outcome in patients undergoing reversal of 
Hartmann's procedures: a multicentre study. Colorectal Dis 2015;17:242-9.

12. Horesh N, Rudnicki Y, Dreznik Y, Zbar AP, Gutman M, Zmora O, et al. Reversal of Hartmann's procedure: still a complicated operation. Tech Coloproctol 2018;22:81-7.

13. Dindo D, Demartines N, Clavien PA. Classification of surgical complications: a new proposal with evaluation in a cohort of 6336 patients and results of a survey. Ann Surg 2004;240:20513.

14. Sugimoto K, Sato K, Maekawa H, Sakurada M, Orita H, Ito T, et al. Analysis of the efficacy of direct hemoperfusion with polymyxin B-immobilized fiber (PMX-DHP) according to the prognostic factors in patients with colorectal perforation. Surg Today 2013;43:1031-8.

15. Alvarez JA, Baldonedo RF, Bear IG, Otero J, Pire G, Alvarez P, et al. Outcome and prognostic factors of morbidity and mortality in perforated sigmoid diverticulitis. Int Surg 2009;94:240-8.

16. Komatsu S, Shimomatsuya T, Nakajima M, Amaya H, Kobuchi T, Shiraishi S, et al. Prognostic factors and scoring system for survival in colonic perforation. Hepatogastroenterology 2005; 52:761-4.

17. Kriwanek S, Armbruster C, Beckerhinn P, Dittrich K. Prognostic factors for survival in colonic perforation. Int J Colorectal Dis 1994;9:158-62.

18. Nakamura F, Yui R, Muratsu A, Sakuramoto K, Muroya T, Ikegawa $\mathrm{H}$, et al. Study of the prognostic factor of the colon perfora- tion case with the pan-peritonitis that needed emergency surgery: a single-center observational study. Acute Med Surg 2019; 6:379-84.

19. Jobin SP, Maitra S, Baidya DK, Subramaniam R, Prasad G, Seenu V. Role of serial lactate measurement to predict 28-day mortality in patients undergoing emergency laparotomy for perforation peritonitis: prospective observational study. J Intensive Care 2019;7:58.

20. Tridente A, Clarke GM, Walden A, McKechnie S, Hutton P, Mills GH, et al. Patients with faecal peritonitis admitted to European intensive care units: an epidemiological survey of the GenOSept cohort. Intensive Care Med 2014;40:202-10.

21. Tan KK, Hong CC, Zhang J, Liu JZ, Sim R. Surgery for perforated colorectal malignancy in an Asian population: an institution's experience over 5 years. Int J Colorectal Dis 2010;25:989-95.

22. Yoo RN, Kye BH, Kim G, Kim HJ, Cho HM. Mortality risk factor analysis in colonic perforation: would retroperitoneal contamination increase mortality in colonic perforation? Ann Surg Treat Res 2017;93:203-8.

23. Strobel O, Werner J, Büchler MW. Surgical therapy of peritonitis. Chirurg 2011;82:242-8.

24. Otani K, Kawai K, Hata K, Tanaka T, Nishikawa T, Sasaki K, et al. Colon cancer with perforation. Surg Today 2019;49:15-20.

25. Halim H, Askari A, Nunn R, Hollingshead J. Primary resection anastomosis versus Hartmann's procedure in Hinchey III and IV diverticulitis. World J Emerg Surg 2019;14:32. 